\title{
Alanyl-glutamine improves pancreatic $\beta$-cell function following ex vivo inflammatory challenge
}

\author{
Vinicius Fernandes Cruzat', Kevin Noel Keane', Anita Lavarda Scheinpflug', \\ Robson Cordeiro', Mario J Soares ${ }^{2}$ and Philip Newsholme ${ }^{1}$
}

${ }^{1}$ School of Biomedical Sciences, and ${ }^{2}$ Directorate of Nutrition, Dietetics and Food Technology, School of Public Health, Curtin Health Innovation Research Institute of Ageing and Chronic Disease - Curtin University, GPO Box U1987, Perth, Western Australia, Australia 6845
Correspondence should be addressed to V F Cruzat Email vinifc@usp.br or vinicius.cruzat@curtin.edu.au

\begin{abstract}
Obesity-associated diabetes and concomitant inflammation may compromise pancreatic $\beta$-cell integrity and function. L-glutamine and L-alanine are potent insulin secretagogues, with antioxidant and cytoprotective properties. Herein, we studied whether the dipeptide L-alanylL-glutamine (Ala-GIn) could exert protective effects via sirtuin 1/HUR (SIRT1/HUR) signalling in $\beta$-cells, against detrimental responses following ex vivo stimulation with inflammatory mediators derived from macrophages (IMMs). The macrophages were derived from blood obtained from obese subjects. Macrophages were exposed (or not) to lipopolysaccharide (LPS) to generate a pro-inflammatory cytokine cocktail. The cytokine profile was determined following analysis by flow cytometry. Insulin-secreting BRIN-BD11 $\beta$-cells were exposed to IMMs and then cultured with or without Ala-GIn for $24 \mathrm{~h}$. Chronic insulin secretion, the L-glutamineglutathione (GSH) axis, and the level of insulin receptor $\beta$ (IR- $\beta$ ), heat shock protein 70 (HSP70), SIRT1/HUR, CCAAT-enhancer-binding protein homologous protein (CHOP) and cytochrome $c$ oxidase IV (COXIV) were evaluated. Concentrations of cytokines, including interleukin 1 $\beta$ (IL1 $\beta$ ), IL6, IL10 and tumour necrosis factor alpha (TNF $\alpha$ ) in the IMMs, were higher following exposure to LPS. Subsequently, when $\beta$-cells were exposed to IMMs, chronic insulin secretion, and IR- $\beta$ and COX IV levels were decreased, but these effects were partially or fully attenuated by the addition of Ala-GIn. The glutamine-GSH axis and HSP70 levels, which were compromised by IMMs, were also restored by Ala-GIn, possibly due to protection of SIRT1/HUR levels, and a reduction of $\mathrm{CHOP}$ expression. Using an ex vivo inflammatory approach, we have demonstrated Ala-Gln-dependent $\beta$-cell protection mediated by coordinated effects on the glutamine-GSH axis, and the HSP pathway, maintenance of mitochondrial metabolism and stimulus-secretion coupling essential for insulin release.
\end{abstract}

\section{Key Words}

- diabetes
- glutamine

- alanine

- GSH

- HSP

- sirtuin 1
Journal of Endocrinology (2015) 224, 261-271

\section{Introduction}

The prevalence of diabetes, especially type 2 diabetes mellitus (T2DM), is continuing to rise at rapid rates (Whiting et al. 2011). In turn, obesity and metabolic syndrome are significant risk predictors for T2DM (Pradhan 2007). The conditions that underscore T2DM include a chronic pro-inflammatory state that, together with the adverse effects of hyperglycaemia and hyperlipidaemia, leads to progressive mitochondrial and pancreatic $\beta$-cell dysfunction (Akude et al. 2011, Kim et al. 2014). Chronic inflammation is characterised by 
enhanced cytokine levels (e.g. interleukin $1 \beta$ (IL1 $\beta)$, IL6, IL10 and tumour necrosis factor alpha (TNF $\alpha)$ ), and oxygen free radicals generated by immune cells, such as activated macrophages (Nackiewicz et al. 2014).

While many amino acids can potentially affect $\beta$-cell integrity, a relatively small number enhance insulin secretion in the presence of glucose; these include L-glutamine and L-alanine (Cunningham et al. 2005, Newsholme et al. 2014). Both amino acids are also important for the synthesis of the tripeptide $\mathrm{L}-\gamma$-glutamylL-cysteinylglycine (GSH, i.e. a major component of the L-glutamine-GSH axis; Cunningham et al. 2005, Cruzat et al. 2014a, Petry et al. 2014). GSH has many protective functions in cellular metabolism, as well as attenuation of oxidative stress and inflammation under catabolic situations (Cruzat \& Tirapegui 2009, Cruzat et al. 2014b). Furthermore, L-glutamine is a potent modulator of the heat shock protein (HSP) response, possibly through the activation of the glucosamine biosynthetic pathway (HBP) and nutrient sensors such as sirtuin 1 (SIRT1) (Newsholme et al. 2014). Interestingly, in T2DM patients, the concentration of the most abundant amino acid in the body, L-glutamine, may be reduced (approximately 20\%), when compared with concentrations in healthy subjects (Menge et al. 2010, Tsai et al. 2012). In addition, in fatty diabetic animal models, the gluconeogenic amino acids, which include L-glutamine, are progressively reduced in plasma, liver and skeletal muscle (Wijekoon et al. 2004).

On the other hand, SIRT1 downstream pathways may be suppressed in chronic inflammatory situations, contributing to chronic low-grade inflammation and impaired insulin secretion (Luu et al. 2013). Hence, L-glutamine together with L-alanine supplied as the L-alanyl-L-glutamine dipeptide (Ala-Gln) may benefit the antioxidant system, attenuating inflammation, and may modulate the HSP response in catabolic situations (Cruzat et al. 2014a,b, Newsholme et al. 2014). A metabolic hallmark of diabetes is the aberrant alteration of glucose and lipid concentrations in the blood. Amino acids including glutamine (decreased) and branched-chain amino acids (increased) are known to be altered in diabetes, but the effects of Ala-Gln have never been tested on $\beta$-cells in the presence or absence of inflammatory mediators, which can be obtained from macrophages following stimulation in vitro (IMMs). The monocytes/macrophages used in this study were isolated from obese humans. Our novel experimental model was designed to provide an ex vivo pancreatic islet environment that mimics the effects of obesity-associated inflammation on $\beta$-cell dysfunction in vivo.

\section{Materials and methods}

\section{Stimulation of macrophages isolated from blood, to produce inflammatory factors}

This study was conducted using macrophages circulating in the blood from 20 obese participants (eight men and 12 women, age $39 \pm 13$, BMI $36.5 \pm 4.6 \mathrm{~kg} / \mathrm{m}^{2}$, body fat $49.3 \pm 5.6 \%$ and HOMA-IR $3.12 \pm 1.33$ ). Dual-energy $\mathrm{X}$-ray absorptiometry (Prodigy model, Lunar Radiation Corporation, Madison, WI, USA) was used to evaluate body composition. Plasma glucose and insulin were analysed by PathWest Laboratory, Royal Perth Hospital. All the procedures were approved by the Human Research Ethics Committee of Curtin University, Perth, Western Australia (protocol number HR 108/2013).

For the purposes of this research, the blood was collected in EDTA tubes and the peripheral blood mononuclear cells (PBMCs, a mixture of monocytes and lymphocytes) and neutrophils isolated using Histopaque 1077 (Sigma-Aldrich). After collection, blood was diluted in 2 mM EDTA PBS (1:2), and this suspension was layered onto 1:1 Histopaque and then centrifuged for $20 \mathrm{~min}$ at $600 \mathrm{~g}$ at room temperature. The PBMCs were collected from the interphase and washed three times with PBS and centrifuged for $10 \mathrm{~min}$ each at 300,200 and $120 \mathrm{~g}$ at room temperature. The PBMCs were maintained in DMEM without phenol red (Life Technologies), supplemented with $10 \%(\mathrm{v} / \mathrm{v})$ FCS, $0.1 \%$ antibiotics $(100 \mathrm{U} / \mathrm{ml}$ penicillin and $0.1 \mathrm{mg} / \mathrm{ml}$ streptomycin), $5.56 \mathrm{mM}$ D-glucose (pH 7.4), $110 \mathrm{mg} / \mathrm{l}$ sodium pyruvate, and $2 \mathrm{mM}$ of Ala-Gln (SigmaAldrich). To allow the adherence of monocytes onto the 24-well plates, the PBMCs were maintained for $3 \mathrm{~h}$ in a humidified atmosphere of $5 \% \mathrm{CO}_{2}$ and $95 \%$ air at $37{ }^{\circ} \mathrm{C}$.

Afterwards, the supernatant medium containing lymphocytes (approximately 98\%) was removed. After washing with PBS, the adherent monocytes in the 24-well plate $(2.0 \times$ $10^{6}$ cells/well) were exposed to DMEM culture medium with and without $1 \mathrm{mM}$ of lipopolysaccharide (LPS from Escherichia coli, strain 0111:B4, Sigma-Aldrich). After 3 h of stimulation, the media were removed and the adhered cells were washed with PBS. The monocytes/macrophages were subsequently incubated for a further $24 \mathrm{~h}$ in DMEM culture medium to obtain IMM - (macrophages not exposed to LPS) and IMM + (macrophages exposed to LPS, see Fig. 1), and stored at $-80^{\circ} \mathrm{C}$ for subsequent experiments.

\section{Culture and treatment of $\beta$-cells}

In this study, clonal insulin-secreting BRIN-BD11 pancreatic $\beta$-cells were utilised, on the basis of their well-characterised

Published by Bioscientifica Ltd 


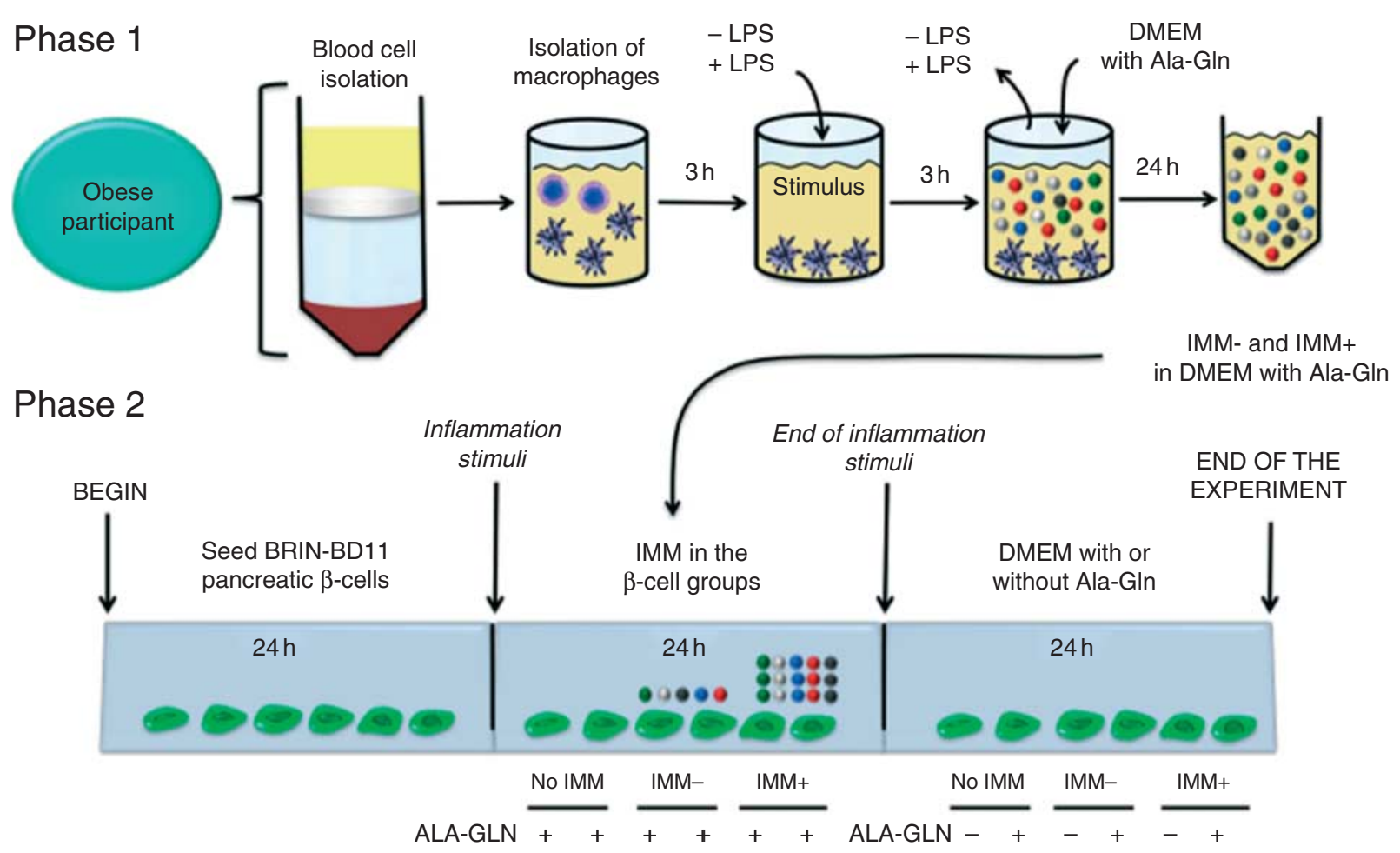

\section{Figure 1}

Schematic of the full experimental design. In the first phase, to obtain the inflammatory mediators from macrophages (IMMs) isolated from obese participants, immune cells were stimulated with (IMM+) or without LPS (IMM -) in DMEM culture media, all in the presence of Ala-GIn. In the second phase, $\beta$-cells were seeded and groups exposed to the DMEM culture media containing IMM - or IMM + for $24 \mathrm{~h}$. After the inflammatory

responses to glucose and amino acids, as well as their reproducible secretory responses, consistent receptor/ signalling protein levels and cell survival/death responses (Cunningham etal. 2005, Corless et al. 2006, Kiely et al. 2007, McClenaghan 2007, Krause et al. 2011, 2014). After culture in vented $75-\mathrm{cm}^{2}$ flasks, cells were seeded in 24 -well plates $\left(1.0 \times 10^{5}\right.$ cells/well $)$ and maintained for $24 \mathrm{~h}$ in a humidified cell culture incubator with $5 \% \mathrm{CO}_{2}$ and $95 \%$ air at $37^{\circ} \mathrm{C}$. After this period, $\beta$-cells were exposed for $24 \mathrm{~h}$ to DMEM (Life Technologies) supplemented with $2 \mathrm{mM}$ Ala-Gln containing IMM - or IMM+. This period was chosen because chronic inflammation has been implicated in $\beta$-cell dysfunction typical of T2DM (Krause et al. 2011). After inflammatory challenge, cells were washed with PBS and maintained for $24 \mathrm{~h}$ in DMEM supplemented or not with Ala-Gln.

Cells not receiving IMMs (no IMM groups), but incubated under the same conditions in DMEM with or without Ala-Gln for a $24 \mathrm{~h}$ period, served as non-stimulated groups for comparative purposes. For each parameter, the experiment was repeated at least four times. A detailed experimental design and flow diagram is provided in Fig. 1. stimulation period, cells were maintained for further $24 \mathrm{~h}$ in IMM-free DMEM media but with and without $2 \mathrm{mM}$ Ala-Gln. Only in this last $24 \mathrm{~h}$ period, cells not receiving IMM (no IMM) were incubated under the same conditions with and without Ala-Gln for comparison purposes with the groups receiving IMM - or IMM + .

\section{Quantification of specific inflammatory factors in the IMM using flow cytometric analyses}

The inflammatory cytokines in the DMEM containing IMM - and IMM + (Fig. 2) were determined using flow cytometric analysis performed on an Attune Acoustic Focusing Cytometer (FACS, Applied Biosystems). Briefly, after LPS stimulation, macrophage supernatant was incubated for 15 min with $50 \mu$ lof an antibody mixture against specific cytokines. Samples were prepared and stained for flow cytometric analysis using the antibodies for IL1 $\beta$, IL6, IL8, IL10 and TNF $\alpha$ (BD Cytometric Bead Array, CBA, San Jose, CA, USA). Data were analysed using the FACS Software FlowJo (TreeStar, Ashland OR, USA).

\section{Measurement of chronic insulin secretion by $\beta$-cells}

Following the last $24 \mathrm{~h}$ period, an aliquot of the medium was removed for insulin assay and quickly stored at $-80{ }^{\circ} \mathrm{C}$ for determination later. Insulin was measured

Published by Bioscientifica Ltd 

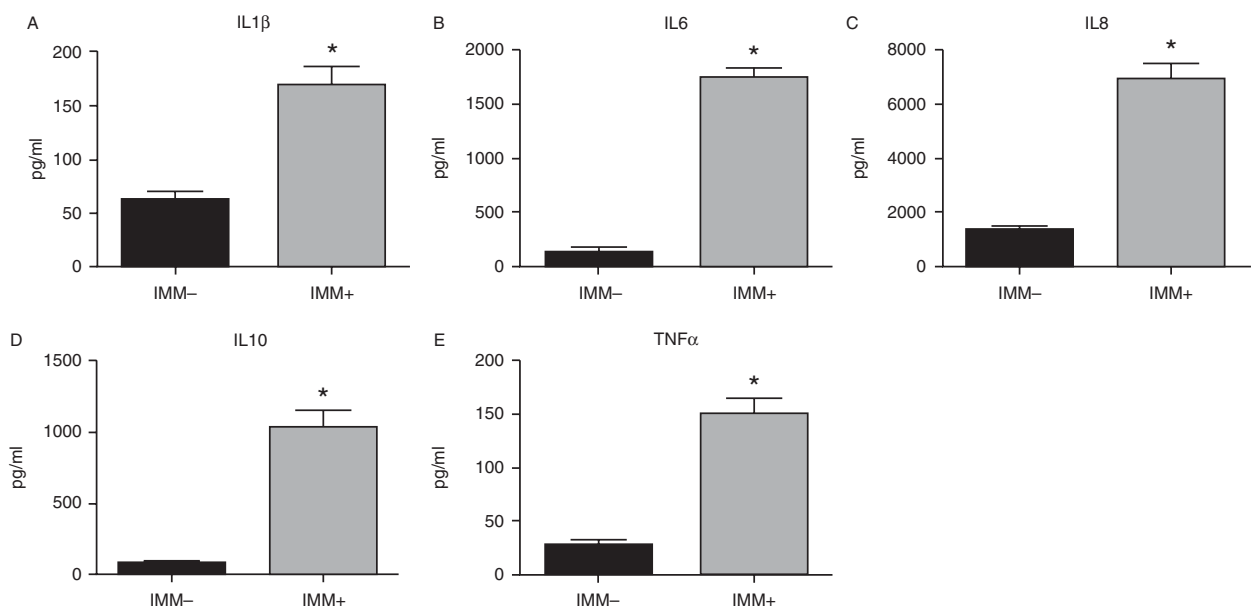

Figure 2

IMM content determined by flow cytometric analysis. Macrophages in 24-well plates $\left(2.0 \times 10^{6}\right.$ cells/well) were exposed or not to LPS for $3 \mathrm{~h}$ (IMM + and IMM - respectively), then washed and maintained for further $24 \mathrm{~h}$ in DMEM culture medium with Ala-Gln to generate the

using the Mercodia Ultrasensitive Rat Insulin ELISA Kit (Mercodia, Uppsala, Sweden).

\section{Cell viability measurements}

Following the experimental procedures described earlier, media were removed, and cells were washed with PBS and stained with Neutral Red $(100 \mu \mathrm{g} / \mathrm{ml}$ of PBS). After $4 \mathrm{~h}$ of incubation, neutral red solution was removed and extracted with acid ethanol (alcohol/glacial acetic acid, 50:1 v/v). The absorbance was measured at a wavelength of $540 \mathrm{~nm}$ using a spectrophotometer (EnSpire Multimode Plate Reader, PerkinElmer, Waltham, MA, USA). The 3-(4,5-Dimethylthiazol-2-yl)-2,5-Diphenyltetrazolium Bromide (MTT) assay was performed as described by Mosmann (1983), and the absorbance was measured at a wavelength of $550 \mathrm{~nm}$.

\section{Measurement of the L-glutamine-GSH axis and GSH disulphide}

Intracellular L-glutamine and L-glutamate concentrations were determined in $\beta$-cell lysates using a commercial kit (Sigma-Aldrich) as described by Lund (1986). BRINBD11 cells were exposed to various conditions as described earlier in a 96-well plate $\left(8.0 \times 10^{3}\right.$ cells/well) and GSH and GSH disulphide (GSSG) were detected using a luminescence-based system (GSH/GSSG-Glo Assay, Promega), and microplate luminometer (EnSpire Multimode Plate Reader) as described by Akerboom \& Sies (1981). cytokine cocktail. (A) Interleukin $1 \beta$ (IL1 $\beta$ ), (B) IL6, (C) IL8, (D) IL10 and (E) tumour necrosis factor alpha (TNF $\alpha)$. ${ }^{*} P<0.0001$ for the comparison with IMM - .

\section{Western blotting analysis}

Following treatment, BRIN-BD11 cells were lysed in $100 \mu \mathrm{l}$ of RIPA lysis buffer containing protease and phosphatase inhibitors (Cell Signaling Technology, Danvers, MA, USA). Cell lysates were transferred to ice-cold microcentrifuge tubes and centrifuged at $14000 \boldsymbol{g}$ for $10 \mathrm{~min}$ at $4{ }^{\circ} \mathrm{C}$ and stored at $-80^{\circ} \mathrm{C}$. Cellular protein concentrations were quantified using the Direct Detect assay-free sample card and measured in the Direct Detect Infra-red Spectrometer (EMD Millipore, Billerica, MA, USA).

Equal amounts of protein were combined with lithium dodecyl sulphate (LDS) sample buffer and the reducing agent dithiothreitol (DTT, Novex, Life Technologies). Proteins (26 $\mu \mathrm{g} /$ lane) were subjected to SDS-PAGE, using 4-20\% Tris-glycine gels, and transferred onto nitrocellulose membrane using iBlot transfer stacks (Life Technologies). The SNAP i.d. (EMD Millipore) quick immunoblot vacuum system was used for immunological detection and membranes were blocked in 3.0\% (w/v) BSA (Amresco, Solon, $\mathrm{OH}$, USA) in PBS-Tween (PBST, 1\% w/v) buffer. Membranes were incubated for $10 \mathrm{~min}$ with the primary antibodies SIRT1 (1:1000), HSP70 (1:1000), cytochrome $c$ oxidase subunit IV (COX IV, 1:1000), insulin receptor $\beta$ and pro-insulin receptor (IR- $\beta$ and PRO-IR respectively, 1:1000), CCAATenhancer-binding protein homologous protein (CHOP, 1:500) and ELAVL1/HUR (1:300). The anti-rabbit IgG (Agilent's Dako, Glostrup, Denmark) diluted 1:2000 was used as a secondary antibody. An anti-rabbit $\beta$-actin (1:1000) was used as a control for protein loading. All of the primary

Published by Bioscientifica Ltd 
antibodies were obtained from Cell Signaling Technology. Membranes were visualised with Clarity Western ECL substrate (Bio-Rad) in the Molecular Imager Gel Doc XR System (Bio-Rad).

\section{Statistical analysis}

Statistical differences between the IMM levels in the DMEM culture media were detected using the two-tailed unpaired parametric $t$-test $(P<0.0001)$. Following confirmation of a normal distribution using KolmogorovSmirnov test, $\beta$-cell results were subjected to ANOVA (one way). Whenever $P$ values were $\leq 0.05$, post-hoc tests employed the multiple comparison procedure of Tukey (Tukey Honestly Significant Difference (HSD)). All statistical calculations and graphics were performed using the GraphPad Prism Software v. 6.0 (La Jolla, CA, USA).

\section{Results}

\section{Identification of IMMs isolated from obese humans}

Once isolated from obese human blood, monocytes/ macrophages were incubated in the presence or absence of LPS for $3 \mathrm{~h}$, and then incubated for a further $24 \mathrm{~h}$ in DMEM with $2 \mathrm{mM}$ Ala-Gln to allow for increased cytokine secretion before determination of the composition of IMMs. As depicted in Fig. 2, all cytokine levels (IL1 $\beta$, IL6, IL8, IL10 and TNF $\alpha)$ were higher $(P<0.0001)$ in the IMM+ group (LPS-stimulated), when compared with the IMM - group, which may mimic the status of inflammation during the progression of T2DM. In this phase of the experimental protocol, both IMM - and IMM+ cells were generated in DMEM supplemented with Ala-Gln.

\section{Effect of IMMs on $\beta$-cell integrity and function}

Inflammation of the islet is associated with the pathophysiology of diabetes. However, the majority of the in vitro studies utilise synthetic cytokine cocktails for cell stimulation. This study represents a novel approach, i.e. challenging $\beta$-cells with inflammatory mediators obtained from ex vivo macrophages (IMMs). L-glutamine and L-alanine are crucial for insulin secretion and can be consumed at high rates by both islets and $\beta$-cells. As depicted in Fig. 3A, the absence per se of $2 \mathrm{mM}$ Ala-Gln in the culture media of $\beta$-cells dramatically decreased chronic (24h) insulin secretion (by 78\%) in the noninflammatory-stimulated (no IMM) groups $(P<0.05)$. A similar effect was observed in cells exposed to both
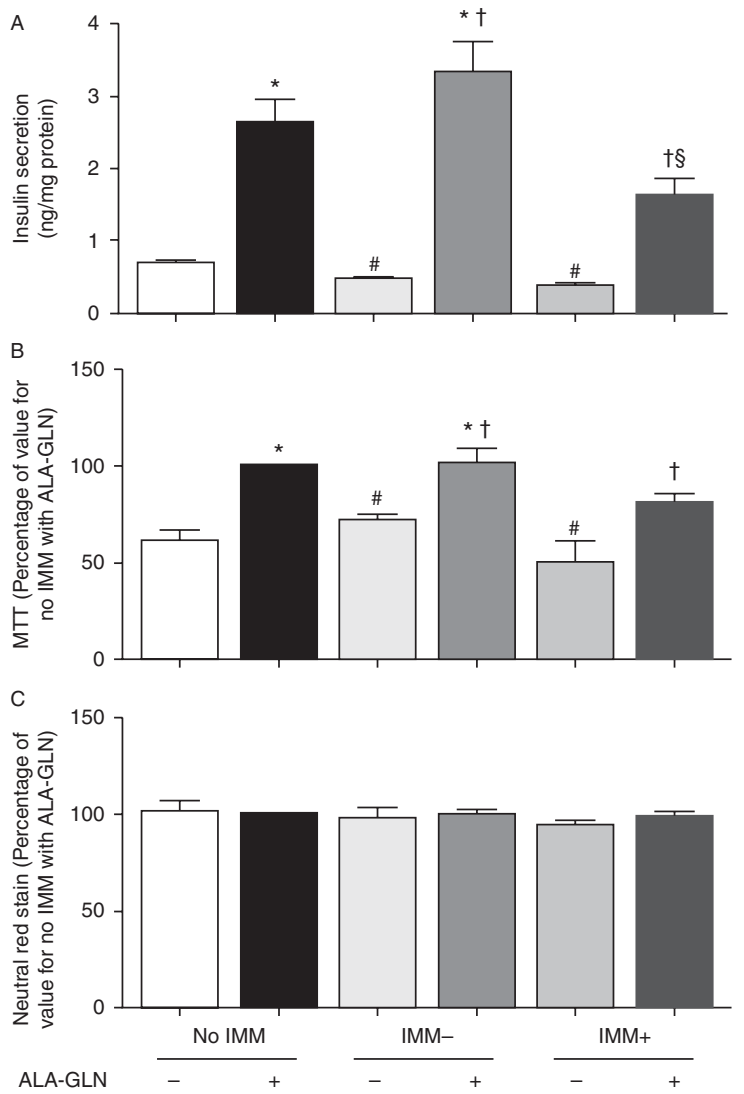

Figure 3

Insulin secretion (A), cellular metabolic activity measured via MTT assay (B) and viability, based on the ability of cells to incorporate and bind the dye neutral red in lysosomes (C) of $\beta$-cells. Data are expressed as mean \pm S.E.M. ${ }^{*} P<0.05$ for the comparison with no IMM group without Ala-GIn. ${ }^{\#} P<0.05$ for the comparison with no IMM with Ala-GIn. ${ }^{\dagger} P<0.05$ Ala-Gln effects on both IMM - and IMM + groups. ${ }^{\S} P<0.05$ for the comparison of IMM - and $\mathrm{IMM}+$, both with Ala-GIn.

IMM - and IMM + in the media without Ala-Gln during the last $24 \mathrm{~h}$. Insulin secretion was 80 and $87 \%$ lower, respectively, when compared with the no IMM group incubated with Ala-Gln $(P<0.05)$. Interestingly, the reduction in insulin secretion from both groups of BRIN-BD11 exposed to both IMMs was attenuated by the availability of Ala-Gln $(P<0.05)$. However, this effect was more pronounced (approximately 50\% higher) in the IMM - group than in the IMM+ group $(P<0.05)$. Moreover, in the IMM - group with Ala-Gln, the insulin secretion was also significantly $(P<0.05)$ higher $(4.5$-fold $)$ when compared with the no IMM group without the dipeptide (Fig. 3A).

Cell metabolic viability was reduced by $37 \%$ in the IMM-absent group without Ala-Gln, when compared with the IMM-absent group with Ala-Gln $(P<0.05)$, indicating the importance of Ala-Gln for cell metabolism and

Published by Bioscientifica Ltd 
Table 1 Glutamine, glutamate, GSH and GSSG content of BRIN-BD11 pancreatic $\beta$-cells following IMM treatment and the effects of L-alanyl-L-glutamine (Ala-GIn). Data are presented as mean \pm s.E.M.

\begin{tabular}{|c|c|c|c|c|c|c|}
\hline \multirow[b]{3}{*}{ Variables } & \multicolumn{6}{|c|}{ Experimental groups } \\
\hline & \multicolumn{2}{|c|}{ No IMM } & \multicolumn{2}{|c|}{ IMM - } & \multicolumn{2}{|c|}{$\mathrm{IMM}+$} \\
\hline & -Ala-Gln & + Ala-Gln & -Ala-Gln & + Ala-Gln & - Ala-Gln & + Ala-Gln \\
\hline Glutamine (nmol/mg protein) & $8.83 \pm 1.43$ & $12.96 \pm 0.51 *$ & $2.70 \pm 1.05^{*^{\dagger}}$ & $10.22 \pm 0.86^{\ddagger}$ & $2.63 \pm 0.50 *^{\dagger}$ & $8.43 \pm 1.26^{\ddagger}$ \\
\hline Glutamate (nmol/mg protein) & $2.03 \pm 0.24$ & $2.34 \pm 0.32$ & $0.83 \pm 0.18$ & $2.55 \pm 0.57^{\ddagger}$ & $0.86 \pm 0.37$ & $2.51 \pm 0.27^{\ddagger}$ \\
\hline GSH $(\mu \mathrm{M})$ & $1.85 \pm 0.15$ & $4.15 \pm 0.14 *$ & $2.39 \pm 0.05^{\dagger}$ & $4.19 \pm 0.17^{*}$ & $2.50 \pm 0.22^{\dagger}$ & $3.63 \pm 0.26^{*}$ \\
\hline GSSG $(\mu \mathrm{M})$ & $0.41 \pm 0.04$ & $0.37 \pm 0.02$ & $0.29 \pm 0.02$ & $0.31 \pm 0.02$ & $0.27 \pm 0.01$ & $0.27 \pm 0.01$ \\
\hline GSH/GSSG & $5.06 \pm 0.49$ & $11.63 \pm 1.01 *$ & $7.95 \pm 0.45^{\dagger}$ & $12.08 \pm 0.62^{*^{\ddagger}}$ & $8.05 \pm 0.49^{\dagger}$ & $13.36 \pm 0.80^{*}$ \\
\hline
\end{tabular}

${ }^{\star} P<0.05$ for the comparison with no IMM group without Ala-GIn. ${ }^{\dagger} P<0.05$ for the comparison with no IMM with Ala-GIn. ${ }^{\ddagger} P<0.05$ Ala-Gln effects on both IMM - and IMM + groups.

function (Fig. 3B). Moreover, in the absence of Ala-Gln, IMM - and IMM + exposure decreased viability as assessed by MTT (by 28 and $48 \%, P<0.05$ ), when compared with the IMM-absent group but in the presence of dipeptide. Nevertheless, the cellular metabolic activity in both groups of BRIN-BD11 cells exposed to IMMs was partially restored (by 30\%) by the presence of Ala-Gln $(P<0.05)$. When compared with the IMM-absent group without Ala-Gln, viability levels as assessed by MTT were higher $(P<0.05)$ in the Ala-Gln exposed BRIN-BD11 cells exposed to IMM - media (Fig. 3B). No differences were observed in the quantitative estimation of cell numbers by neutral red uptake assay (Fig. 3C).

\section{The importance of the $\beta$-cell L-glutamine-GSH axis and redox state}

Ala-GIn effects in no IMM groups Although inflammation is involved in $\beta$-cell dysfunction, a reduction per se in the availability of L-glutamine and L-alanine, both important L-glutamate precursors required for GSH synthesis, may make $\beta$-cells vulnerable to oxidative stress. Indeed, L-glutamine concentrations in no IMM groups was decreased in the absence of Ala-Gln (by 32\%, $P<0.05$, Table 1). This affected the availability of GSH, which was lowered by $55 \%$ in the group without Ala-Gln $(P<0.05)$. While no differences were observed in the GSSG content, the GSH-to-GSSG ratio (GSH/GSSG), an index of intracellular redox status was 56\% lower in the absence of Ala-Gln $(P<0.05$, Table 1$)$. These results indicate that in the absence of Ala-Gln, a harmful intracellular oxidative environment was promoted.

\section{Effects of IMMs and evidence of Ala-GIn protection} BRIN-BD11 cells in the absence of Ala-Gln but exposed to both IMM - and IMM+ were associated with reduced
L-glutamine concentration, by almost $70 \%$, when compared with both IMM-absent groups $(P<0.05$, Table 1). This effect lowered GSH concentration (by approximately 40\%) and GSH/GSSG ratio (by approximately $30 \%$ ), observed in $\beta$-cell groups exposed to IMMs, when compared with the no IMM group with Ala-Gln $(P<0.05)$.

On the other hand, the addition of Ala-Gln to the $\beta$-cells was able to restore L-glutamine and L-glutamate levels in both groups exposed to IMMs $(P<0.05)$. Moreover, in both IMM groups, the pro-oxidative situation was reversed $(P<0.05)$ by the addition of the dipeptide to the $\beta$-cells, as assessed by GSH content (increased by $31 \%$ ) and the GSH/GSSG ratio (by almost 34\%). This GSH response was also higher $(P<0.05)$ than that in the IMM-absent group without Ala-Gln (Table 1).

\section{Effects of IMMs and Ala-GIn on protein expression of $\beta$-cells}

The exposure of $\beta$-cells exposure to both IMM - and $\mathrm{IMM}+$ reduced IR- $\beta$ levels by approximately $60 \%$ $(P<0.05$; Fig. 4C), in the absence of Ala-Gln, when compared with the IMM-absent group, supplemented with Ala-Gln. However, the availability of the dipeptide in both IMM-treated groups reversed this scenario by increasing IR- $\beta$, and this effect was much greater $(P<0.05)$ in the IMM+ group with Ala-Gln, compared with the IMM-absent group incubated without Ala-Gln. Conversely, the presence of Ala-Gln lowered the expression of PRO-IR by 58\% (Fig. 4A) in the IMM-absent group, and at least $45 \%$ in BRIN-BD11 cells exposed to both IMMs, when compared with the IMM-absent group incubated without Ala-Gln $(P<0.05)$. Lower PRO-IR levels were also observed in cells exposed to IMM - and IMM + and incubated with Ala-Gln compared with the respective

Published by Bioscientifica Ltd. 

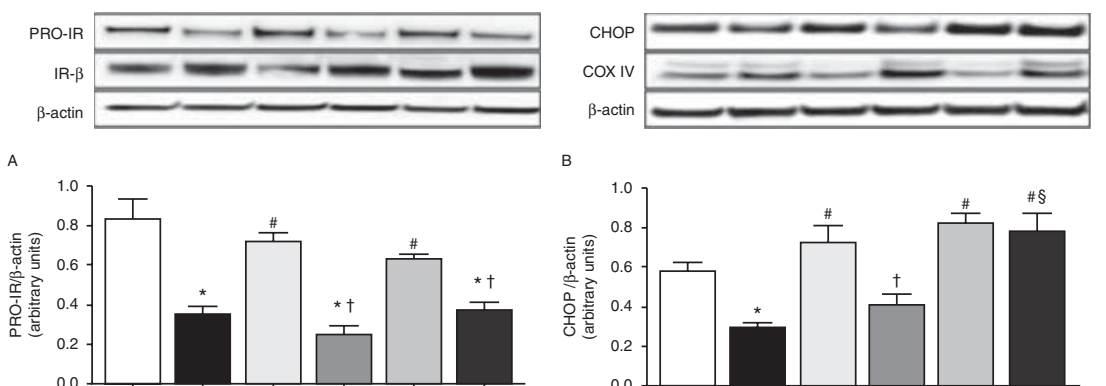

B
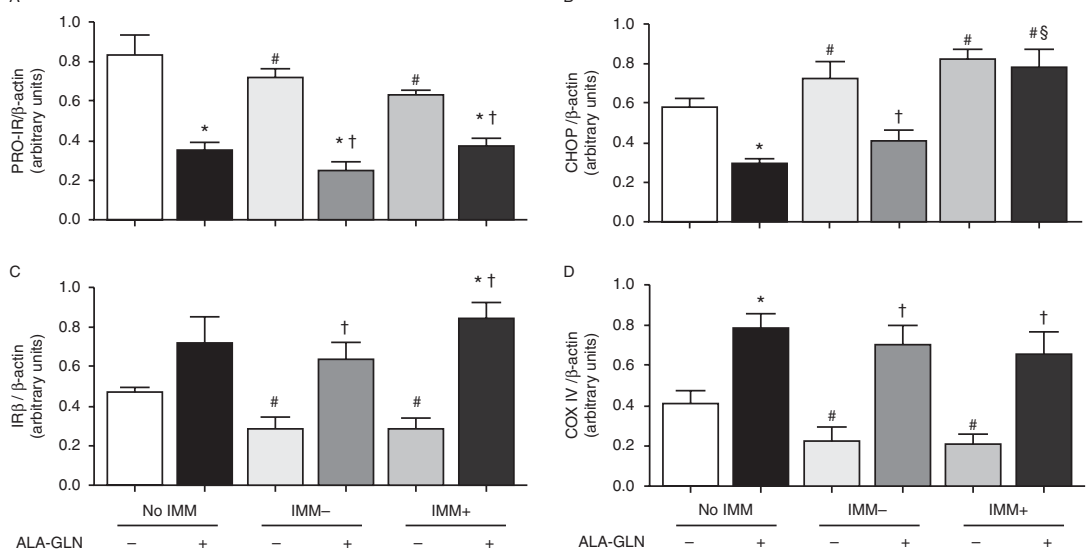

Figure 4

PRO-IR (A), CHOP (B), IR- $\beta(C)$, and COX IV (D) protein levels obtained from BRIN-BD11 $\beta$-cells following IMM challenge with or without Ala-GIn were measured by Western blot analysis. Data are expressed as mean \pm s.E.M. ${ }^{\star} P<0.05$ for the comparison with no IMM group without Ala-GIn. ${ }^{\#} P<0.05$

groups without Ala-Gln. Moreover, both IMM-treated groups without Ala-Gln presented higher PRO-IR expression, when compared with the IMM-absent group incubated with dipeptide $(P<0.05)$.

As expected, the absence of Ala-Gln per se increased CHOP expression (by almost 50\%), a marker of endoplasmic reticulum (ER) stress, in the IMM-absent groups $(P<0.05$, Fig. 4B). The same effect was observed in the groups exposed to both IMM - and IMM + in cells incubated without Ala-Gln $(P<0.05)$. Interestingly, when Ala-Gln was added to the $\beta$-cells exposed to IMM-, CHOP levels were lower (by 42\%) than those in the corresponding group incubated without Ala-Gln $(P<0.05)$. Despite this effect, the CHOP level was still elevated (by almost 45\%) in the IMM + group incubated with Ala-Gln, when compared with the IMM-absent group incubated with Ala-Gln $(P<0.05)$, possibly a consequence of the high content of cytokines in the IMM+ group. Furthermore, COX IV levels were lower (almost 53\%) in all groups of BRIN-BD11 cells incubated in the absence of Ala-Gln $(P<0.05$, Fig. 4D). Conversely, incubation in the presence of Ala-Gln reversed this scenario $(P<0.05)$, thus maintaining COX IV levels.

The nutrient-dependent modulators, SIRT1, HUR and HSP70 (the major HSP), were reduced (by 55, 66 and 75\% respectively) in the IMM-absent group incubated without Ala-Gln, when compared with the same conditions with the dipeptide ( $P<0.05$, Fig. 5A, $\mathrm{C}$ and $\mathrm{B}$ respectively). for the comparison with no IMM with Ala-GIn. ${ }^{\dagger} P<0.05$ Ala-GIn effects on both IMM - and IMM + groups. ${ }^{\S} P<0.05$ for the comparison of IMM - and $\mathrm{IMM}+$, both with Ala-GIn.

Furthermore, in cells incubated without Ala-Gln, but exposed to both IMM - and IMM+, almost 50\% lower expression of these proteins occurred, compared with the IMM-absent group incubated with Ala-Gln $(P<0.05)$. However, in the presence of both IMM - and IMM+, addition of Ala-Gln to the $\beta$-cells reversed this scenario $(P<0.05)$, by increasing SIRT1, HUR and HSP70 levels, compared with cells incubated without Ala-Gln $(P<0.05)$. The summary of the beneficial effects of Ala-Gln on $\beta$-cell function and protein expression following inflammatory challenge is provided in Table 2.

\section{Discussion}

Inflammation is an adaptive and protective response of multicellular organisms against infections. On the other hand, chronic metabolic diseases, such as obesity and T2DM, are characterised by meta-inflammation, wherein elevation of several pro-inflammatory cytokines induces changes in metabolic functioning and homoeostatic set points that may underscore the progression of obesity to diabetes (Medzhitov 2010). The objective of this study was to try and mimic the inflammatory milieu in vivo not only to understand how $\beta$-cell function and integrity were affected by a low or high dose of cytokines, but also to discover whether the dipeptide Ala-Gln had protective effects with respect to insulin secretion, signal transduction and other functional parameters affected

Published by Bioscientifica Ltd 

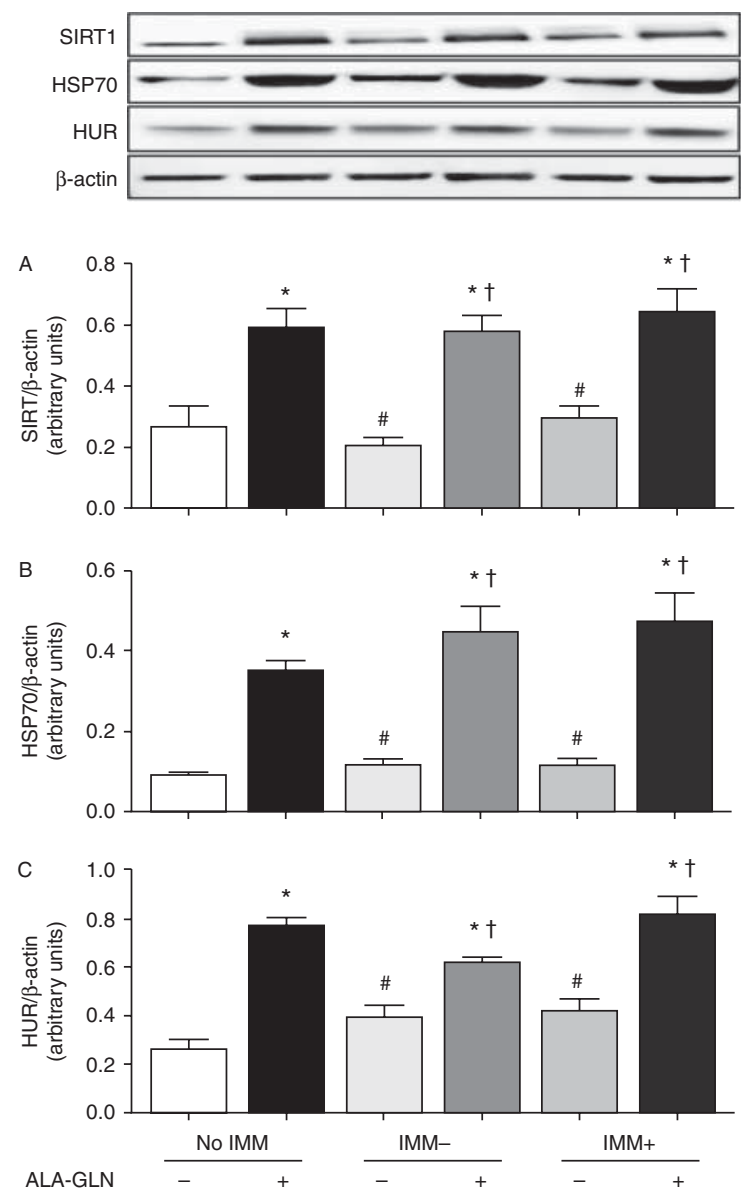

\section{Figure 5}

SIRT1 (A), HSP70 (B) and HUR (C) protein levels obtained from BRIN-BD11 $\beta$-cells following IMM challenge with or without Ala-Gln were measured by Western blot analysis. Data are expressed as mean \pm s.E.M. ${ }^{*} P<0.05$ for the comparison with the no IMM group without Ala-GIn. ${ }^{\#} P<0.05$ for the comparison with the no IMM group with Ala-Gln. ${ }^{\dagger} P<0.05$ Ala-Gln effects on both IMM - and IMM + groups.

by these cytokines. Using a novel approach, where monocytes/macrophages were isolated from obese patients recruited in our clinic and used to generate a 'cocktail' of inflammatory cytokines ex-vivo in the basal (unstimulated) state or after stimulation with LPS, subsequent functional effects of the cytokine cocktail on $\beta$-cells were then compared with a control treatment, where $\beta$-cells were not exposed to cytokines (as shown in Fig. 1). The effects of the cytokine cocktail (IMMs) on $\beta$-cell function and integrity have never been studied before, to the best of our knowledge. However, we recognise the limitations of our study, in that we did not isolate monocytes/macrophages from lean human volunteers and we have used a rat clonal $\beta$-cell line rather than human cells, as no equivalent human $\beta$-cell line (in terms of magnitude of metabolic, signalling and secretory responses) is currently available. However, it has been reported previously that the major human proinflammatory cytokine, IL1 $\beta$, elicited potent suppression of insulin secretion in both human and rat islet cells (Kawahara \& Kenney 1991).

As expected, when the monocytes/macrophages obtained from obese participants were exposed to a proinflammatory stimulant (LPS), the release of IL1 $\beta$, IL6, IL8, IL10 and TNF $\alpha$ increased significantly. In response to inflammation, the $\beta$-cell undergoes protective changes, that enhance survival but at the expense of insulin secretion (Kahn et al. 2006, Richardson et al. 2009, Jourdan et al. 2013). In these scenarios, L-glutamine is particularly important for ensuring adequate levels of protection and insulin secretion (Corless et al. 2006). In this study, the availability of L-glutamine through the addition of Ala-Gln resulted in attenuation of the negative effects of IMM exposure, with respect to insulin secretion (Fig. 3A). These results are in agreement with results from previous studies (Corless et al. 2006), in which L-glutamine availability for BRIN-BD11 cells resulted in optimisation of insulin secretion and was associated with up-regulation of 148 genes by at least 1.8-fold and down-regulation of 18 genes related to insulin signalling and metabolism.

Recent reports have described reductions in circulating levels of L-glutamine in diabetes (Etgen \& Oldham 2000, Wijekoon et al. 2004, Menge et al. 2010, Tsai et al. 2012). The absence of $2 \mathrm{mM}$ Ala-Gln lowered cellular L-glutamine levels and compromised the GSH concentration in the $\beta$-cells as described herein (Table 1). This study does not reveal whether the observed beneficial effects of Ala-Gln are mediated by L-glutamine or L-alanine. However, it has been previously reported that both amino acids are precursors of L-glutamate for de novo GSH generation (Rutten et al. 2005, Cruzat \& Tirapegui 2009), providing protection against oxidative

Table 2 The beneficial effects of incubation in the presence of Ala-GIn (compared with absence) on $\beta$-cell function and protein expression following an inflammatory challenge

\begin{tabular}{|c|c|c|}
\hline Parameters & IMM - & IMM + \\
\hline Insulin secretion & $\uparrow \uparrow$ & $\uparrow$ \\
\hline MTT & $\uparrow$ & $\uparrow$ \\
\hline IR- $\beta$ & $\uparrow$ & $\uparrow$ \\
\hline PRO-IR & $\downarrow$ & $\downarrow$ \\
\hline HSP70 & $\uparrow$ & $\uparrow$ \\
\hline SIRT1 & $\uparrow$ & $\uparrow$ \\
\hline HUR & $\uparrow$ & $\uparrow$ \\
\hline CHOP & $\downarrow$ & $\uparrow \uparrow$ \\
\hline COX IV & $\uparrow$ & $\uparrow$ \\
\hline
\end{tabular}

http://joe.endocrinology-journals.org DOI: 10.1530/JOE-14-0677
(C) 2015 Society for Endocrinology Printed in Great Britain 
stress and inflammation (Cruzat et al. 2010, 2014a, Krause et al. 2011, Petry et al. 2014). GSH acts as a scavenger system and has the ability to counteract reactive oxygen species (ROS, e.g. hydrogen peroxide) by reducing peroxides, protecting cell membranes and reducing lipid peroxidation. As a cause or consequence, oxidative stress has been suggested to play an integral part in the pathogenesis of various organ dysfunctions and disease (Pradhan 2007, Newsholme et al. 2009, Krause et al. 2014).

GSH redox coupling systems are critical to $\beta$-cell integrity and function, as these cells express low levels of ROS-metabolising enzymes, such as catalase and glutathione peroxidase (Tiedge et al. 1997, Takahashi et al. 2014). The GSH oxidation ratio (GSH redox potential $\left.\mathrm{E}_{\mathrm{GSH}}\right)$ is an indicator of the balance between protein thiol oxidation and the ability to reduce them with GSH making use of NADPH as an electron donor to convert GSSG back to GSH (Cruzat \& Tirapegui 2009, Newsholme et al. 2009). In fact, without the presence of Ala-Gln, the GSH/GSSG ratio was low under all conditions tested (Table 1 ), which makes the $\beta$-cells more vulnerable to oxidative stress. However, we have demonstrated herein that Ala-GIn supplementation in the media reinforces the substrate availability for maintenance of the GSH/GSSG ratio, favouring a reduced state. This finding can be particularly important, as excessive production of ROS alters the function of voltage-dependent channels, such as ATP-sensitive $\mathrm{K}^{+}$-channels, electron transport chain proteins and essential components for insulin exocytosis (Newsholme et al. 2009, Krause et al. 2011). The prooxidant state promoted by both IMMs decreased IR- $\beta$ levels, possibly due to the lack of insulin secretion (i.e. autocrine/paracrine effect), which also promoted an inversed PRO-IR concentration (Fig. 4C and A respectively). However, the presence of Ala-Gln reversed this scenario.

Mitochondrial oxidative stress and the subsequent effects on ATP synthesis are one of the most important mechanisms responsible for a reduction in insulin secretion in T2DM. COX IV is required to catalyse the reduction of molecular oxygen to water coupled to the translocation of protons across the mitochondrial inner membrane to the matrix, which drives ATP synthesis. Diabetic animal models (Akude et al. 2011) and other inflammatory scenarios display lower COX IV expression and decreased respiratory chain activity (Hahn et al. 2014, Krause et al. 2014). In turn, this contributes to abnormalities in cellular energy metabolism and a lowering of insulin secretion (Akude et al. 2011, Hahn et al. 2014). Our results are in agreement with these findings, as the absence of Ala-Gln per se, and exposure to IMM+ and IMM - decreased COX IV levels (Fig. 4D).

In order to protect the cell against diverse environmental and stress-inducing situations, such as ischaemia, inflammation and oxidative stress, the HSP response is an organised conservative genetic mechanism that acts as a molecular chaperone, preventing the accumulation of misfolded proteins. Moreover, expression of HSP is now widely accepted as anti-inflammatory in action, as it blocks the cyclic activation and nuclear binding of nuclear factor $\kappa \mathrm{B}(\mathrm{NF} \kappa \mathrm{B})$ to the promoters of inflammatory genes that could otherwise compromise cell integrity and function. Thus, HSP attenuates unresolved inflammation (Newsholme \& de Bittencourt 2014). Amino acids, such as L-glutamine, are required for the optimal HSP70 response, through the HBP-induced activation of SIRT1/HUR (Lafontaine-Lacasse et al. 2011, Newsholme et al. 2014), which was observed in our study (Fig. 5A and C respectively). Enhanced HUR may promote the stability of numerous target mRNAs, including that encoding SIRT1, via association of HUR with the $3^{\prime}$-untranslated region of Sirt1 mRNA, that promotes its stability and thus a rise in Sirt1 expression (Newsholme \& de Bittencourt 2014). In turn, SIRT1 enhances the expression of the main heat shock factor, HSF1, and heat shock elements (HSEs) in the nuclei, resulting in expression of HSP70 (Kotas et al. 2013, Cruzat et al. 2014a, Petry et al. 2014), as observed herein (Fig. 5B).

In contrast, SIRT1 can suppress inflammation and stimulate HSP responses; however, in response to chronic inflammation, similar to that in obesity-associated diabetes, the SIRT1/HUR axis is repressed (Kotas et al. 2013), contributing to the unresolved inflammation (LafontaineLacasse et al. 2011, Luu et al. 2013). In this study, using our in vitro model to mimic in vivo inflammation, we observed that Ala-Gln maintained the HSP70 levels via the SIRT1/HUR axis. This is a protective effect as reduced levels of HSP70 in obesity-associated diabetes are correlated with reduced insulin sensitivity, hyperglycaemia and hyperinsulinaemia (Chung et al. 2008). Moreover, inflammation in obesity is related to ER stress (Hummasti \& Hotamisligil 2010), and this in turn, plays a central role in the development of insulin resistance and diabetes (Ozcan et al. 2006). In response to such conditions, cells react to ER dysfunction by increasing the expression of CHOP, which has observed in our study (Fig. 4B). Interestingly, the absence of Ala-Gln per se enhanced CHOP, indicating an important role for amino acids in the control of ER stress. Some reports have described increased levels of CHOP in insulitis (Marhfour et al. 2012) and depletion of

Published by Bioscientifica Ltd 
GSH (Kim et al. 2008), which increases the production of ROS in the ER. However, our results indicated that even with increased levels of $\mathrm{CHOP}, \mathrm{GSH}$ was restored in the Ala-Gln groups with and without IMM.

Although significant progress has been made in understanding the pathogenesis of diabetes, the prevalence of the condition is continuously rising and will challenge health care systems worldwide in the 21st century. Many of the underlying molecular mechanisms are still unknown, and consequently new effective treatments are elusive. In this study, we have provided evidence for the importance of amino acids and related dipeptides, e.g. Ala-Gln, for $\beta$-cell integrity and function following inflammatory challenge (Table 2). The effects observed herein are mediated via intermediary metabolism, mitochondrial function, and antioxidant and HSP responses. Our work may have implications for development of novel in vivo approaches, with the aim of protecting $\beta$-cell function.

\section{Declaration of interest}

The authors declare that there is no conflict of interest that could be perceived as prejudicing the impartiality of the research reported.

\section{Funding}

V F C was in receipt of postdoctoral fellowship from the Brazilian National Council for Scientific and Technological Development (CNPq - Science Without Borders Programme, process 245562/2012-5).

\section{Author contribution statement \\ This work was designed by V F C, K N K and P N. The initial manuscript and drafts were prepared by V F C and revised by V F C, K N K and P N. Patients were recruited and the measurements were carried out in the laboratory of M J S. Body composition data were obtained by M J S. All experiments and biochemical analyses were performed by V F C and K N K. A L S and R C contributed to western blot analysis of IR- $\beta$ and PRO-IR protein levels. Data and statistical analyses and figure preparation were performed by V F C. Supervision of the experimental work and manuscript preparation were preformed by $\mathrm{P} \mathrm{N}$ and $\mathrm{M} J \mathrm{~S}$. All authors approved the final version of the paper.}

\section{Acknowledgements}

The authors thank Curtin University School of Biomedical Sciences and the School of Public Health and Faculty of Health Sciences for research support. They also thank Prof. Paulo Ivo Homem de Bittencourt Jr and Ms Graziella Nicoletti for the discussions and interest.

\section{References}

Akerboom TP \& Sies H 1981 Assay of glutathione, glutathione disulfide, and glutathione mixed disulfides in biological samples. Methods in Enzymology 77 373-382. (doi:10.1016/S0076-6879(81)77050-2)
Akude E, Zherebitskaya E, Chowdhury SK, Smith DR, Dobrowsky RT \& Fernyhough P 2011 Diminished superoxide generation is associated with respiratory chain dysfunction and changes in the mitochondrial proteome of sensory neurons from diabetic rats. Diabetes 60 288-297. (doi:10.2337/db10-0818)

Chung J, Nguyen AK, Henstridge DC, Holmes AG, Chan MH, Mesa JL, Lancaster GI, Southgate RJ, Bruce CR, Duffy SJ et al. 2008 HSP72 protects against obesity-induced insulin resistance. PNAS 105 1739-1744. (doi:10.1073/pnas.0705799105)

Corless M, Kiely A, McClenaghan NH, Flatt PR \& Newsholme P 2006 Glutamine regulates expression of key transcription factor, signal transduction, metabolic gene, and protein expression in a clonal pancreatic $\beta$-cell line. Journal of Endocrinology 190 719-727. (doi:10.1677/joe.1.06892)

Cruzat VF \& Tirapegui J 2009 Effects of oral supplementation with glutamine and alanyl-glutamine on glutamine, glutamate, and glutathione status in trained rats and subjected to long-duration exercise. Nutrition 25 428-435. (doi:10.1016/j.nut.2008.09.014)

Cruzat VF, Rogero MM \& Tirapegui J 2010 Effects of supplementation with free glutamine and the dipeptide alanyl-glutamine on parameters of muscle damage and inflammation in rats submitted to prolonged exercise. Cell Biochemistry and Function 28 24-30. (doi:10.1002/cbf. 1611)

Cruzat VF, Pantaleao LC, Donato J Jr, de Bittencourt PI Jr \& Tirapegui J $2014 a$ Oral supplementations with free and dipeptide forms of L-glutamine in endotoxemic mice: effects on muscle glutamineglutathione axis and heat shock proteins. Journal of Nutritional Biochemistry 25 345-352. (doi:10.1016/j.jnutbio.2013.11.009)

Cruzat VF, Bittencourt A, Scomazzon SP, Leite JS, de Bittencourt PI Jr \& Tirapegui J 2014b Oral free and dipeptide forms of glutamine supplementation attenuate oxidative stress and inflammation induced by endotoxemia. Nutrition 30 602-611. (doi:10.1016/j.nut. 2013.10.019)

Cunningham GA, McClenaghan NH, Flatt PR \& Newsholme P 2005 L-Alanine induces changes in metabolic and signal transduction gene expression in a clonal rat pancreatic $\beta$-cell line and protects from pro-inflammatory cytokine-induced apoptosis. Clinical Science $\mathbf{1 0 9}$ 447-455. (doi:10.1042/CS20050149)

Etgen GJ \& Oldham BA 2000 Profiling of Zucker diabetic fatty rats in their progression to the overt diabetic state. Metabolism 49 684-688. (doi:10.1016/S0026-0495(00)80049-9)

Hahn WS, Kuzmicic J, Burrill JS, Donoghue MA, Foncea R, Jensen MD, Lavandero S, Arriaga EA \& Bernlohr DA 2014 Proinflammatory cytokines differentially regulate adipocyte mitochondrial metabolism, oxidative stress, and dynamics. American Journal of Physiology. Endocrinology and Metabolism 306 E1033-E1045. (doi:10.1152/ajpendo. 00422.2013)

Hummasti S \& Hotamisligil GS 2010 Endoplasmic reticulum stress and inflammation in obesity and diabetes. Circulation Research $\mathbf{1 0 7}$ 579-591. (doi:10.1161/CIRCRESAHA.110.225698)

Jourdan T, Godlewski G, Cinar R, Bertola A, Szanda G, Liu J, Tam J, Han T, Mukhopadhyay B, Skarulis MC et al. 2013 Activation of the Nlrp3 inflammasome in infiltrating macrophages by endocannabinoids mediates $\beta$ cell loss in type 2 diabetes. Nature Medicine 19 1132-1140. (doi:10.1038/nm.3265)

Kahn SE, Hull RL \& Utzschneider KM 2006 Mechanisms linking obesity to insulin resistance and type 2 diabetes. Nature $\mathbf{4 4 4} 840-846$. (doi:10.1038/nature05482)

Kawahara DJ \& Kenney JS 1991 Species differences in human and rat islet sensitivity to human cytokines. Monoclonal anti-interleukin-1 (IL-1) influences on direct and indirect IL-1-mediated islet effects. Cytokine 3 117-124. (doi:10.1016/1043-4666(91)90031-8)

Kiely A, McClenaghan NH, Flatt PR \& Newsholme P 2007 Pro-inflammatory cytokines increase glucose, alanine and triacylglycerol utilization but inhibit insulin secretion in a clonal pancreatic $\beta$-cell line. Journal of Endocrinology 195 113-123. (doi:10.1677/JOE-07-0306) 
Kim I, Xu W \& Reed JC 2008 Cell death and endoplasmic reticulum stress: disease relevance and therapeutic opportunities. Nature Reviews. Drug Discovery 7 1013-1030. (doi:10.1038/nrd2755)

Kim C, Patel P, Gouvin LM, Brown ML, Khalil A, Henchey EM, Heuck AP \& Yadava N 2014 Comparative analysis of the mitochondrial physiology of pancreatic $\beta$ cells. Bioenergetics 3 110. (doi:10.4172/2167-7662. 1000110)

Kotas ME, Gorecki MC \& Gillum MP 2013 Sirtuin-1 is a nutrient-dependent modulator of inflammation. Adipocyte 2 113-118. (doi:10.4161/adip. 23437)

Krause MS, McClenaghan NH, Flatt PR, de Bittencourt PI, Murphy C \& Newsholme P 2011 L-Arginine is essential for pancreatic $\beta$-cell functional integrity, metabolism and defense from inflammatory challenge. Journal of Endocrinology 211 87-97. (doi:10.1530/JOE-11-0236)

Krause M, Keane K, Rodrigues-Krause J, Crognale D, Egan B, De Vito G, Murphy C \& Newsholme P 2014 Elevated levels of extracellular heat-shock protein 72 (eHSP72) are positively correlated with insulin resistance in vivo and cause pancreatic $\beta$-cell dysfunction and death in vitro. Clinical Science 126 739-752. (doi:10.1042/CS20130678)

Lafontaine-Lacasse M, Dore G \& Picard F 2011 Hexosamines stimulate apoptosis by altering SIRT1 action and levels in rodent pancreatic $\beta$-cells. Journal of Endocrinology 208 41-49. (doi:10.1677/JOE-10-0243)

Lund P 1986 L-Glutamine and L-glutamate: UV-method with glutaminase and glutamate dehydrogenase. In Methods of Enzymatic Analysis, 1st edn, pp 357-363. Ed HU Bergmeyer. Weinheim, Germany: Verlagsgesellschaft.

Luu L, Dai FF, Prentice KJ, Huang X, Hardy AB, Hansen JB, Liu Y, Joseph JW $\&$ Wheeler MB 2013 The loss of Sirt1 in mouse pancreatic $\beta$ cells impairs insulin secretion by disrupting glucose sensing. Diabetologia 56 2010-2020. (doi:10.1007/s00125-013-2946-5)

Marhfour I, Lopez XM, Lefkaditis D, Salmon I, Allagnat F, Richardson SJ Morgan NG \& Eizirik DL 2012 Expression of endoplasmic reticulum stress markers in the islets of patients with type 1 diabetes. Diabetologia 55 2417-2420. (doi:10.1007/s00125-012-2604-3)

McClenaghan NH 2007 Physiological regulation of the pancreatic $\beta$-cell: functional insights for understanding and therapy of diabetes. Experimental Physiology 92 481-496. (doi:10.1113/expphysiol.2006.034835)

Medzhitov R 2010 Inflammation 2010: new adventures of an old flame. Cell 140 771-776. (doi:10.1016/j.cell.2010.03.006)

Menge BA, Schrader H, Ritter PR, Ellrichmann M, Uhl W, Schmidt WE \& Meier JJ 2010 Selective amino acid deficiency in patients with impaired glucose tolerance and type 2 diabetes. Regulatory Peptides 160 75-80. (doi:10.1016/j.regpep.2009.08.001)

Mosmann T 1983 Rapid colorimetric assay for cellular growth and survival: application to proliferation and cytotoxicity assays. Journal of Immunological Methods 65 55-63. (doi:10.1016/0022-1759(83)90303-4)

Nackiewicz D, Dan M, He W, Kim R, Salmi A, Rutti S, Westwell-Roper C, Cunningham A, Speck M, Schuster-Klein C et al. 2014 TLR2/6 and TLR4-activated macrophages contribute to islet inflammation and impair $\beta$ cell insulin gene expression via IL-1 and IL-6. Diabetologia 57 1645-1654. (doi:10.1007/s00125-014-3249-1)
Newsholme P \& de Bittencourt PI Jr 2014 The fat cell senescence hypothesis: a mechanism responsible for abrogating the resolution of inflammation in chronic disease. Current Opinion in Clinical Nutrition and Metabolic Care 17 295-305. (doi:10.1097/MCO. 0000000000000077)

Newsholme P, Morgan D, Rebelato E, Oliveira-Emilio HC, Procopio J, Curi R \& Carpinelli A 2009 Insights into the critical role of NADPH oxidase(s) in the normal and dysregulated pancreatic $\beta$ cell. Diabetologia 52 2489-2498. (doi:10.1007/s00125-009-1536-z)

Newsholme P, Cruzat V, Arfuso F \& Keane K 2014 Nutrient regulation of insulin secretion and action. Journal of Endocrinology 221 R105-R120. (doi:10.1530/JOE-13-0616)

Ozcan U, Yilmaz E, Ozcan L, Furuhashi M, Vaillancourt E, Smith RO, Gorgun CZ \& Hotamisligil GS 2006 Chemical chaperones reduce ER stress and restore glucose homeostasis in a mouse model of type 2 diabetes. Science 313 1137-1140. (doi:10.1126/science.1128294)

Petry ER, Cruzat VF, Heck TG, Leite JS, Homem de Bittencourt PI Jr \& Tirapegui J 2014 Alanyl-glutamine and glutamine plus alanine supplements improve skeletal redox status in trained rats: involvement of heat shock protein pathways. Life Sciences 94 130-136. (doi:10.1016/ j.lfs.2013.11.009)

Pradhan A 2007 Obesity, metabolic syndrome, and type 2 diabetes: inflammatory basis of glucose metabolic disorders. Nutrition Reviews 65 S152-S156. (doi:10.1301/nr.2007.dec.S152-S156)

Richardson SJ, Willcox A, Bone AJ, Foulis AK \& Morgan NG 2009 Isletassociated macrophages in type 2 diabetes. Diabetologia 52 1686-1688. (doi:10.1007/s00125-009-1410-z)

Rutten EP, Engelen MP, Schols AM \& Deutz NE 2005 Skeletal muscle glutamate metabolism in health and disease: state of the art. Current Opinion in Clinical Nutrition and Metabolic Care 8 41-51. (doi:10.1097/ 00075197-200501000-00007)

Takahashi HK, Santos LR, Roma LP, Duprez J, Broca C, Wojtusciszyn A \& Jonas JC 2014 Acute nutrient regulation of the mitochondrial glutathione redox state in pancreatic $\beta$-cells. Biochemical Journal 460 411-423. (doi:10.1042/BJ20131361)

Tiedge M, Lortz S, Drinkgern J \& Lenzen S 1997 Relation between antioxidant enzyme gene expression and antioxidative defense status of insulin-producing cells. Diabetes 46 1733-1742. (doi:10.2337/diab. 46.11.1733)

Tsai PH, Yeh CL, Liu JJ, Chiu WC \& Yeh SL 2012 Effects of dietary glutamine on inflammatory mediator gene expressions in rats with streptozotocin-induced diabetes. Nutrition 28 288-293. (doi:10.1016/ j.nut.2011.06.003)

Whiting DR, Guariguata L, Weil C \& Shaw J 2011 IDF diabetes atlas: global estimates of the prevalence of diabetes for 2011 and 2030. Diabetes Research and Clinical Practice 94 311-321. (doi:10.1016/j.diabres.2011. 10.029)

Wijekoon EP, Skinner C, Brosnan ME \& Brosnan JT 2004 Amino acid metabolism in the Zucker diabetic fatty rat: effects of insulin resistance and of type 2 diabetes. Canadian Journal of Physiology and Pharmacology 82 506-514. (doi:10.1139/y04-067)

Received in final form 19 December 2014

Accepted 23 December 2014

Accepted Preprint published online 23 December 2014 http://joe.endocrinology-journals.org DOI: 10.1530/JOE-14-0677
C 2015 Society for Endocrinology Printed in Great Britain
Published by Bioscientifica Ltd. 